\title{
Constraining Parity Violation in Gravity with Measurements of Neutron-Star Moments of Inertia
}

\author{
Nicolás Yunes, ${ }^{1}$ Dimitrios Psaltis, ${ }^{2,3}$ Feryal Özel, ${ }^{2,3}$ and Abraham Loeb ${ }^{3}$ \\ ${ }^{1}$ Department of Physics, Princeton University, Princeton, NJ 08544, USA. \\ ${ }^{2}$ Department of Astronomy, University of Arizona, Tucson, AZ 85721, USA. \\ ${ }^{3}$ Institute for Theory and Computation (ITC), Harvard Smithsonian \\ Center for Astrophysics, 60 Garden St., Cambridge, MA 02138, USA.
}

(Dated: September 6, 2021)

\begin{abstract}
Neutron stars are sensitive laboratories for testing general relativity, especially when considering deviations where velocities are relativistic and gravitational fields are strong. One such deviation is described by dynamical, Chern-Simons modified gravity, where the Einstein-Hilbert action is modified through the addition of the gravitational parity-violating Pontryagin density coupled to a field. This four-dimensional effective theory arises naturally both in perturbative and non-perturbative string theory, loop quantum gravity, and generic effective field theory expansions. We calculate here Chern-Simons modifications to the properties and gravitational fields of slowly spinning neutron stars. We find that the Chern-Simons correction affects only the gravitomagnetic sector of the metric to leading order, thus introducing modifications to the moment of inertia but not to the mass-radius relation. We show that an observational determination of the moment of inertia to an accuracy of $10 \%$, as is expected from near-future observations of the double pulsar, will place a constraint on the Chern-Simons coupling constant of $\xi^{1 / 4} \lesssim 5 \mathrm{~km}$, which is at least three-orders of magnitude stronger than the previous strongest bound.

PACS numbers: 04.40.Dg,04.50.Kd,04.60.Rt,04.80.Cc
\end{abstract}

\section{INTRODUCTION}

Even though it has been almost a century since its original proposal, general relativity (GR) remains only marginally tested in the strong, dynamical regime, where velocities are relativistic and gravitational fields are strong. Great effort has gone into testing this theory in the solar system and with the binary pulsar (via the so-called parameterized post-Newtonian [1 6] and postKeplerian frameworks $[6-8]$ ), as well as in cosmological settings (e.g., via the so-called parameterized postFriedmannian framework 9-12]). With the imminent discovery of gravitational waves, new frameworks have been proposed (e.g., the parameterized post-Einstenian one 13 or the multipole expansion of black-hole spacetimes [14 17]) that will allow us to search for GR deviations with gravitational waves in the strong, dynamical regime. Until such observations become available, however, one must rely on the next best tool to test GR in the strong field: observations of neutron stars and black holes in the electromagnetic spectrum (see e.g., Ref. [18, 19]).

GR tests with neutron stars appear a priori to be not as clean as solar system or binary pulsar tests. When dealing with weak-field systems, we can usually employ the effacement principle [20, 21] to treat objects as effective point particles moving under the influence of some force, which mathematically resembles Maxwell's electromagnetism (the so-called gravitomagnetic analogy; see eg. 22]). In modified theories of gravity, however, the effacement principle and Birkhoff's theorem need not hold, but corrections usually arise at first order in weak-field perturbation theory (see e.g., [6] for a review of internal structure effects in alternative theories).
When dealing with neutron stars, we must inescapably account for the matter content of the star properly, as this can greatly influence the astrophysical observables to leading order. Our lack of detailed knowledge of their matter content is encoded in the equation of state (EOS), which relates the matter density to its pressure. Several EOSs have been studied in the literature (see eg. [23, 24]), differing mostly in the nature of the strong interaction at very high densities and the presence of a softening component (such as hyperons, Bose condensates or quark matter), none of which we possess laboratory data for. Different possible EOSs thus lead to slightly different electromagnetic observables, which generically mimic alternative theory effects. Neutron star tests of GR must thus address possible degeneracies between the EOS and true GR deviations.

Such a degeneracy between matter content and gravitational theories is not new to GR tests. For example, the observed acceleration of the universe can either be attributed to a dark energy fluid or to modifications to the Einstein equations. This degeneracy in the explanation of observables is a consequence of the Strong Equivalence Principle, which essentially states that it is difficult to differentiate between a gravitational field and a matter distribution, since they are tied together by Einstein's equations.

The only way to break this degeneracy is to possess at least two independent sets of neutron star observations: one to determine the EOS as a function of the theory under consideration and another one to pin down the specific theory. Such two-observation tests have been dramatically successful when testing GR with binary pulsars, where the degeneracies are associated with the un- 
known masses of the pulsars [ [6]. New and exciting observations are beginning to constrain the neutron star EOS [25, 26], suggesting that such two-observation tests will become possible in the near future. In this paper, we consider a particular EOS for neutron-star matter that is consistent with current observations. Our results, however, depend weakly on our ignorance of the EOS. We show that the corrections introduced by the specific alternative theory considered couples strongly only to large density gradients, which occur only close to the neutron star surface, where our knowledge of the EOS is fairly robust.

The construction of modified gravity theories is a nontrivial endevour that can be classified into two groups: infrared modifications and ultraviolet modifications (see eg., [13, 27] for a review). The first group deals with corrections to the action that modify the weak-field predictions of GR. Examples of these include $f(R)$ gravity 28 30], DGP gravity [31], Horava-Lifshitz gravity [32, 33], and $\mathrm{TeVeS}$ 34, 35]. Usually, such modifications are introduced to propose explanations for the late-time expansion of the universe or the anomalous rotation curves of galaxies. The second group corrects the action by introducing higher-order curvature terms, which, by construction, do not modify the leading-order predictions of GR in the weak-field. These theories do modify the strong-field regime of gravity, where neutron star observations can place stringent constraints. Examples include Chern-Simons (CS) modified gravity [36], Gauss-Bonnet modified gravity [37], scalar-tensor theories [38 40], and certain $f(R)$ models 41 44.

We concentrate here on tests of dynamical CS modified gravity, which is currently only weakly constrained by binary pulsar observations [45]. This four-dimensional theory adds a certain contraction of two Riemann tensors and the Levi-Civita tensor to the Einstein-Hilbert action, coupled to a dynamical scalar field. It can be thought of as an effective theory (a low-energy approximation to some more fundamental theory) whose signature is the modification of parity asymmetric gravitational solutions, such as the Kerr spacetime. It naturally arises as the low-energy approximation of many different fundamental theories. The latter could be string theory (i.e., Type I, IIb, Heterotic, etc.), where the inclusion of the CS term is inescapable in the perturbative sector by the Green-Schwarz anomaly-cancelling mechanism [46, 47]. In non-perturbative string theory, CS corrections also arise in the presence of D-instanton charges due to duality symmetries [48]. The more fundamental theory could also be loop quantum gravity, where the CS correction has also been seen to generically arise when one of the fundamental constants of that theory is promoted to a field [47, 49 51]. Even without recurring to a specific fundamental theory, the CS term unavoidably arises in effective field theories as one of the only non-vanishing, second-order curvature corrections to the Einstein-Hilbert action in inflation [52].

The non-dynamical version of CS-modified gravity (where the scalar field is non-evolving, but prescribed a priori) has been extensively studied and greatly constrained in the past [48, 53 68]. Only recently, however, has there been a dedicated effort to analyze the dynamical theory [45, 69 71]. In Ref. [45], a small-coupling approximation was developed to find the exterior gravitational field of a slowly rotating compact object. Perhaps rather surprisingly [72, 73], the solution was found to deviate from the Kerr spacetime because such a spacetime sources a non-vanishing CS correction that couples to the spin-angular momentum of the black hole. Later, in [70], the Strong Equivalence Principle was found to be satisfied in the dynamical theory and point particles were indeed found to follow geodesics of the background spacetime.

In this paper, we calculate the CS-modified gravitational field inside neutron stars and relate this to possible observables that would allow us to constrain the theory. This analysis should be seen as complimentary to that of [45], thus completing the gravitational field prescription everywhere in the spacetime of rotating bodies. Our analysis is also similar to that of [63], except that here we focus on the dynamical theory instead of the nondynamical one and we employ a more realistic, tabulated EOS. We shall see that if we employed the homogeneous stellar structure equation used in [63], the interior solution would not be CS-modified. Although in principle there will be CS modifications to the Dirac equation that describes the motion of fermions inside the star [68], we shall not consider those here.

We find that the main CS correction affects the gravitomagnetic sector of the metric to leading order, as was found for the exterior solution [45]. Such a correction introduces modifications to the moment of inertia of the star, but not to the mass-radius relation to leading order. This implies that the usual degeneracy between alternative theory modifications and the EOS can be broken: measurements of the mass-radius relation from subkilohertz neutron stars can be used to extract the EOS, while measurements of the moment of inertia can be used to test the theory, given the EOS.

Such a degeneracy break is sensitive to the spinfrequency of the neutron star observed. If the neutron star is spinning faster than $\Omega \sim 1 \mathrm{kHz}$, then second-order corrections in the spin-frequency to the mass-radius relation are not necessarily negligible and the degeneracy is not broken. One might then wonder whether such rapidly rotating neutron stars exist in Nature. Up to date, most of the neutron stars observed spin slower than this limit (for a recent modeling of millisecond pulsars see [4] ). However, over the past year there has been a breakthrough in millisecond pulsar searches through combined observations with the latest Fermi data. Such a breakthrough relies on millisecond pulsars generically being strong $\gamma$-ray emitters 75 ]. If so, this pulsar population could be calibrated through the diffuse $\gamma$-ray background they produce [77] (for an outline of such a new survey based on unidentified Fermi sources see [6] ]). The 
tests we describe in this paper need not necessarily apply to this population, as then the degeneracy between mass-radius relation and CS modifications could not be easily broken.

Assuming that the degeneracy can be broken through observations of neutron stars with sub-kHz rotational frequencies, we find that a $10 \%$ accurate, moment of inertia measurement, as is expected in the near future with observations of the double pulsar [78, 79], could place a constraint on the CS coupling constant of $\xi^{1 / 4} \lesssim 5 \mathrm{~km}$. Such a constraint would be three-orders of magnitude stronger than the previous strongest bound [45].

Before proceeding with the remainder of the paper, we first comment on the relationship between neutron-star observations or tests and gravitational wave ones. In the past, these two programs could have been seen as competing approaches, when in reality they should be seen as complementary. Gravitational wave observations sample the fully dynamical, propagating sector of a theory, while neutron star observations test the strong-field, but stationary sector. Moreover, simultaneous gravitational wave-neutron star observations, e.g. of a merging neutron star binary, could yield the most stringent bounds on alternative theories, as the combination of observations would help break degeneracies.

This paper is divided as follows: Sec. [I]defines the basics of CS modified gravity; Sec. III describes the metric parameterization and the stress-energy description of the neutron star; Sec. IV discusses CS modifications to neutron star models; Sec. $\mathrm{V}$ solves the CS modified equations of neutron star structure numerically and present some results; Sec. VI computes some neutron star observables that are CS-modified and discusses possible future constraints on the theory; Sec. VII concludes and points to future research.

We use the following conventions in this paper: we work exclusively in four spacetime dimensions with signature $(-,+,+,+)$ [80]; Latin letters $(a, b, \ldots, h)$ range over all spacetime indices; round and square brackets around indices denote symmetrization and antisymmetrization respectively, namely $T_{(a b)}=\frac{1}{2}\left(T_{a b}+T_{b a}\right)$ and $T_{[a b]}=\frac{1}{2}\left(T_{a b}-T_{b a}\right)$; partial derivatives are sometimes denoted by commas (e.g., $\partial \theta / \partial r=\partial_{r} \theta=\theta_{, r}$ ), while radial derivatives are sometimes denoted with an overhead prime $\partial_{r} \omega=\omega^{\prime}$ if $\omega=\omega(r)$; the notation $A_{(m, n)}$ stands for a term of $\mathcal{O}(m, n)$, which itself stands for terms of $\mathcal{O}\left(\epsilon^{m}\right)$ or $\mathcal{O}\left(\zeta^{n}\right)$; the Einstein summation convention is employed unless otherwise specified; finally, we use geometrized units where $G=c=1$ and where $M_{\odot}=1.476 \mathrm{~km}$ stands for a solar mass.

\section{THE ABC OF CS MODIFIED GRAVITY}

In this section, we review some of the basics of CS modified gravity (see the recent review article [36] for further details).

\section{A. Basic Equations}

The CS action is defined as

$$
S \equiv S_{\mathrm{EH}}+S_{\mathrm{CS}}+S_{\vartheta}+S_{\mathrm{mat}}
$$

where

$$
\begin{aligned}
S_{\mathrm{EH}} & \equiv \kappa \int_{\mathcal{V}} d^{4} x \sqrt{-g} R \\
S_{\mathrm{CS}} & \equiv \frac{\alpha}{4} \int_{\mathcal{V}} d^{4} x \sqrt{-g} \vartheta{ }^{*} R R \\
S_{\vartheta} & \equiv-\frac{1}{2} \int_{\mathcal{V}} d^{4} x \sqrt{-g}\left[g^{a b}\left(\nabla_{a} \vartheta\right)\left(\nabla_{b} \vartheta\right)+2 V(\vartheta)\right] \\
S_{\mathrm{mat}} & \equiv \int_{\mathcal{V}} d^{4} x \sqrt{-g} \mathcal{L}_{\mathrm{mat}}
\end{aligned}
$$

Equation (2) is the standard Einstein-Hilbert term; Equation (3) is the CS correction; Equation (4) is the scalar-field term; Equation (5) describes additional matter sources, with $\mathcal{L}_{\text {mat }}$ the matter Lagrangian density. The quantity $\kappa^{-1} \equiv 16 \pi G$ is the coupling constant of GR, while $\alpha$ is the CS coupling constant. This formulation assumes that the CS scalar field $\vartheta$ is dimensionless, which then forces $\alpha$ to have units of length squared ${ }^{1}$. As usual, $g$ is the determinant of the metric, $\nabla_{a}$ is the covariant derivative associated with the metric tensor $g_{a b}$, $R$ is the Ricci scalar, ${ }^{*} R R$ is the Pontryagin density

$$
{ }^{*} R R=R \tilde{R}={ }^{*} R_{b}^{a}{ }^{c d} R^{b}{ }_{a c d},
$$

and the dual Riemann-tensor is

$$
{ }^{*} R_{b}^{a}{ }^{c d} \equiv \frac{1}{2} \epsilon^{c d e f} R_{b e f}^{a},
$$

with $\epsilon^{\text {cdef }}$ the 4-dimensional Levi-Civita tensor with sign convention $\epsilon^{0123}=+1 / \sqrt{-g}$, where $g$ is the determinant of the metric.

The CS field equations are derived from the variation of the action with respect to the metric tensor and the CS coupling field:

$$
\begin{aligned}
R_{a b}+\frac{\alpha}{\kappa} C_{a b} & =\frac{1}{2 \kappa}\left(T_{a b}-\frac{1}{2} g_{a b} T\right), \\
\square \vartheta & =\frac{d V}{d \vartheta}-\frac{\alpha}{4} * R R,
\end{aligned}
$$

with $R_{a b}$ the Ricci tensor, $\square \equiv \nabla_{a} \nabla^{a}$ the D'Alembertian operator, and $C_{a b}$ the $\mathrm{C}$-tensor

$$
C^{a b} \equiv\left(\nabla_{c} \vartheta\right) \epsilon^{c d e(a} \nabla_{e} R_{d}^{b)}+\left(\nabla_{c} \nabla_{d} \vartheta\right){ }^{*} R^{d(a b) c} .
$$

The total stress-energy tensor is

$$
T_{a b} \equiv T_{a b}^{\mathrm{mat}}+T_{a b}^{\vartheta},
$$

\footnotetext{
1 This formulation is equivalent to that of [45] with the choice $\beta \rightarrow 1$.
} 
where $T_{a b}^{\text {mat }}$ stands for matter contributions, and $T_{a b}^{\vartheta}$ is the scalar field contribution

$$
T_{a b}^{\vartheta} \equiv\left(\nabla_{a} \vartheta\right)\left(\nabla_{b} \vartheta\right)-\frac{1}{2} g_{a b}\left(\nabla_{a} \vartheta\right)\left(\nabla^{a} \vartheta\right)-g_{a b} V(\vartheta)
$$

We set the potential $V(\vartheta)=0$ at the scales of interest, with deviations possibly at the scale of supersymmetry breaking (see e.g., the arguments presented in [36]).

Taking the divergence of Eq. (8), the first term on the left-hand side vanishes by the Bianchi identities, while the second terms on both sides of this equation cancel each other because

$$
\nabla_{a} C^{a b}=-\frac{1}{8}\left(\nabla^{b} \vartheta\right){ }^{*} R R=\frac{1}{2 \kappa} \nabla^{a} T_{a b}^{\vartheta},
$$

provided that Eq. (9) is satisfied. This then establishes that $\nabla_{a} T_{\text {mat }}^{a b}=0$ in dynamical CS modified gravity, thus forcing point particles to move on geodesics.

Two versions of CS modified gravity exist: the nondynamical version and the fully dynamical one. In the former, the scalar field is an a priori, prescribed function that lacks dynamics. With our choice of coupling normalization, this theory can be reproduced in the limit $\alpha \rightarrow \infty$, as then the scalar field evolution equation becomes ${ }^{*} R R=0$ (the so-called Pontryagin constraint), which restricts the space of allowed solutions. We shall not consider this model here, as it has been heavily constrained by solar system and binary pulsar observations [36, 57, 58, 63, 66, 81, 82. Instead, we shall concentrate on the fully dynamical theory, whose equations of motion are given in Eqs. (8) and (9).

\section{B. Perturbative Constraints in Dynamical CS Modified Gravity}

The main idea of perturbative constraints (see e.g., 44, 83 87]) is to perform a small-coupling expansion of the solution to a certain set of differential equations. From a mathematical standpoint, this is nothing but an application of asymptotic analysis or perturbation theory (see e.g., [88]).

In the context of CS modified gravity, perturbative constraints reduce to the small-coupling approximation [45], where one searches for solutions of Eqs. (8) and (9) in the limit $\zeta \ll 1$, where $\zeta$ is of $\mathcal{O}\left[\alpha^{2} /\left(\mathcal{L}^{4} \kappa\right)\right]$ and $\mathcal{L}$ is some characteristic scale that describes the physical system under consideration. For example, when searching for spinning black hole solutions [45], $\mathcal{L}_{\mathrm{BH}}=M_{\mathrm{BH}}$ with $M_{\mathrm{BH}}$ the black hole mass and $\zeta=\alpha^{2} /\left(\kappa M^{4}\right)$. In the case of neutron stars, the natural length scale is the neutron star radius $\mathcal{L}_{\mathrm{NS}}=R$ and $\zeta=\alpha^{2} /\left(\kappa R^{4}\right)$.

In addition to the use of the perturbative constraint scheme (i.e., the small-coupling approximation), we additionally focus on slowly-rotating solutions. We define slow-rotation by requiring that $\epsilon \ll 1$, where $\epsilon=\mathcal{O}(\mathcal{L} \Omega)$ and $\Omega$ is the angular velocity of the physical system.
As before, since for neutron stars $\mathcal{L}_{\mathrm{NS}}=R$, then $\epsilon=$ $\mathcal{O}(R \Omega)$, where $\Omega$ is the star's angular velocity.

The quantities $\epsilon$ and $\zeta$ are book-keeping perturbative parameters associated with the slow-rotation and smallcoupling approximations, respectively. We shall use them to remind ourselves of the order of the approximation. Note that $\zeta \sim \alpha^{2}$ instead of $\sim \alpha$ because the evolution equation for $\vartheta$ forces this field to be linear in $\alpha$, and thus, the $\mathrm{C}$-tensor becomes linear in $\alpha$ as well, leading to corrections to the field equations that scale as $\alpha^{2}$.

Combining the small-coupling and slow-rotation approximations leads to bivariate or two-parameter expansions. Schematically, all fields $A$ are expanded as

$$
A=\sum_{m, n} \epsilon^{m} \zeta^{n} A_{(m, n)},
$$

where $A_{(m, n)}$ is a term of $\mathcal{O}\left(\epsilon^{m}, \zeta^{n}\right)$, which means a term of $\mathcal{O}\left(\epsilon^{m}\right)$ and $\mathcal{O}\left(\zeta^{n}\right)$. For example, a term $A_{(1,1)}$ is proportional to $\epsilon \zeta$ and thus of $\mathcal{O}(\epsilon, \zeta)$, while a term $A_{(2,1)}$ is proportional to $\epsilon^{2} \zeta$ and thus of $\mathcal{O}\left(\epsilon^{2}, \zeta\right)$ [clearly $A_{(2,1)}$ is negligible relative to $\left.A_{(1,1)}\right]$. The metric tensor can be expanded bivariately as

$$
\begin{aligned}
g_{a b} & =g_{a b}^{(0,0)}+\zeta g_{a b}^{(0,1)}+\epsilon g_{a b}^{(1,0)} \\
& +\zeta^{2} g_{a b}^{(0,2)}+\epsilon^{2} g_{a b}^{(2,0)}+\epsilon \zeta g_{a b}^{(1,1)}+\mathcal{O}(3,3)
\end{aligned}
$$

where $g_{a b}^{(0,0)}$ is some background metric that satisfies the Einstein equations, while $\left(g_{a b}^{(0,1)}, g_{a b}^{(1,0)}\right)$ are first order perturbations and $\left(g_{a b}^{(0,2)}, g_{a b}^{(2,0)}, g_{a b}^{(1,1)}\right)$ are second-order perturbations. Given an exact solution to the Einstein equations, $g_{a b}^{\mathrm{GR}}$, its expansion in $\epsilon$ must satisfy

$$
g_{a b}^{\mathrm{GR}}=g_{a b}^{(0,0)}+\epsilon g_{a b}^{(1,0)}+\epsilon^{2} g_{a b}^{(2,0)}+\mathcal{O}\left(\epsilon^{3}\right) .
$$

Thus, all terms $g_{a b}^{(N, 0)}$ for all $N$ are pieces of the slowrotation expansion of a GR solution, while terms that are proportional to $\zeta$ represent CS corrections.

The scalar field can also be expanded bivariately via

$$
\begin{aligned}
\vartheta_{a b} & =\vartheta_{a b}^{(0,0)}+\zeta \vartheta_{a b}^{(0,1)}+\epsilon \vartheta_{a b}^{(1,0)} \\
& +\zeta^{2} \vartheta_{a b}^{(0,2)}+\epsilon^{2} \vartheta_{a b}^{(2,0)}+\epsilon \zeta \vartheta_{a b}^{(1,1)}+\mathcal{O}(3,3)
\end{aligned}
$$

Each piece in this expansion is determined by the evolution equation for the scalar field [Eq. (9)], which in turn depends on the Pontryagin density. In [45, 64], it was shown that for spherically symmetric spacetimes ${ }^{*} R R=0$, thus forcing any physical CS scalar field to a constant and allowing us to set $\vartheta^{(0, N)}=0$ for all $N$. Angular momentum breaks the spherical symmetry and forces ${ }^{*} R R \sim \Omega \sim \epsilon$ to leading order, which then allows us to write

$$
\vartheta_{a b}=\epsilon \vartheta_{a b}^{(1,0)}+\epsilon^{2} \vartheta_{a b}^{(2,0)}+\epsilon \zeta \vartheta_{a b}^{(1,1)}+\mathcal{O}(3,3) .
$$

We see that, to leading order, the C-tensor is linear in $\epsilon$, which then forces the leading order correction to the 
metric to be proportional to $(\epsilon \zeta)$, i.e., a second-order correction in perturbation theory. The metric expansion simplifies to

$$
g_{a b}=g_{a b}^{\mathrm{GR}}+\epsilon \zeta g_{a b}^{(1,1)}+\mathcal{O}(3,3) .
$$

Solving the modified field equations reduces simply to solving for $g_{a b}^{(1,1)}$.

The combination of the small-coupling and slowrotation approximations establishes a well-defined iteration scheme. First, we solve the Einstein equations to linear order in $\epsilon$ to obtain $g_{a b}^{(0,0)}+\epsilon g_{a b}^{(1,0)}$. Second, we use this metric to compute ${ }^{*} R R_{(1,0)}$ and from this solve Eq. (9) for $\vartheta^{(1,0)}$. Third, we use this scalar field and the $g_{a b}^{(0,0)}$ piece of the metric to compute the C-tensor, which when combined with the Einstein tensor $G_{a b}^{(1,1)}$ leads to differential equations for $g_{a b}^{(1,1)}$. Fourth, we solve these differential equations and iterate the scheme to higher order (see, e.g., Ref. [45] for more details on this bootstrapping technique). In this paper, we work to leading order only.

\section{METRIC PARAMETERIZATION AND THE STRESS-ENERGY TENSOR OF NEUTRON-STAR MATTER}

In this section, we consider the parameterization of the metric tensor and the description of the stress-energy tensor for neutron-star matter. We shall search for solutions to the field equations for a metric of the form [89]

$d s^{2}=-e^{2 \lambda} d t^{2}+e^{2 \beta} d r^{2}+r^{2} e^{2 \gamma}\left[d \theta^{2}+e^{2 \chi} \sin ^{2} \theta(d \phi-\omega d t)^{2}\right]$

where $(r, \theta, \phi)$ are (Boyer-Lindquist) spherical polar coordinates and $(\lambda, \beta, \gamma, \chi, \omega)$ are independent functions of radius and polar angle $(r, \theta)$. The function $\omega$ is effectively the angular velocity of an observer falling in from infinity and, as such, is at least linearly proportional to the angular velocity of the rotating star.

We decompose the metric functions in bivariate expansions as

$$
\begin{aligned}
\lambda & =\lambda_{(0,0)}(r)+\epsilon^{2} \lambda_{(2,0)}(r, \theta)+\epsilon \zeta \lambda_{(1,1)}(r, \theta), \\
\beta & =\beta_{(0,0)}(r)+\epsilon^{2} \beta_{(2,0)}(r, \theta)+\epsilon \zeta \beta_{(1,1)}(r, \theta), \\
\gamma & =\gamma_{(0,0)}(r)+\epsilon^{2} \gamma_{(2,0)}(r, \theta)+\epsilon \zeta \gamma_{(1,1)}(r, \theta), \\
\chi & =\chi_{(0,0)}(r)+\epsilon^{2} \chi_{(2,0)}(r, \theta)+\epsilon \zeta \chi_{(1,1)}(r, \theta), \\
\omega & =\epsilon \omega_{(1,0)}(r)+\epsilon \zeta \omega_{(1,1)}(r, \theta),
\end{aligned}
$$

To linear order in $\epsilon$, we can safely assume that all functions of $\mathcal{O}(0,0)$ and $\mathcal{O}(1,0)$ depend only on radius. Notice that we have set $\left(\lambda_{(0,1)}, \beta_{(0,1)}, \gamma_{(0,1)}, \chi_{(0,1)}\right)=0$ and $\left(\lambda_{(0,2)}, \beta_{(0,2)}, \gamma_{(0,2)}, \chi_{(0,2)}\right)=0$ as the C-tensor corrections start at $\mathcal{O}(1,1)$, since there cannot be CSdeformations that are angular momentum independent due to parity invariance. Notice further that we have also set $\left(\omega_{(0,0)}, \omega_{(2,0)}\right)=0$ and $\left(\lambda_{(1,0)}, \beta_{(1,0)}, \gamma_{(1,0)}, \chi_{(1,0)}\right)=0$ because the GR gravitomagnetic and diagonal sectors are known to be series in odd and even powers of the angular velocity respectively in the slow-rotation approximation [89]. Finally, we set $\gamma_{(0,0)}=1=\chi_{(0,0)}$, which amounts to a redefinition of the radial coordinate.

In order to get a feel for how these functions behave, we first present the exterior gravitational field of a neutron star in GR. This exterior field is given by the slowrotation expansion of the Kerr metric, where the smallrotation parameter $\epsilon$ is now of $\mathcal{O}(M a)$, with $M$ the mass of the compact object and $a$ its Kerr parameter. Notice that the Kerr parameter is related to the angular momentum of the compact object via $|J|=M a$, which then implies $a=I \Omega / M$, since by definition $J=I \Omega$, where $I$ is the moment of inertia (to be defined properly later). To zeroth order in the spin, we find

$$
\begin{aligned}
& \left(e^{2 \lambda}\right)_{(0,0)}=1-\frac{2 M}{r}, \quad\left(e^{2 \beta}\right)_{(0,0)}=\left(1-\frac{2 M}{r}\right)^{-1}, \\
& \left(e^{2 \gamma}\right)_{(0,0)}=1, \quad\left(e^{2 \chi}\right)_{(0,0)}=1, \quad \omega_{(0,0)}=0 . \quad(22)
\end{aligned}
$$

To linear order we find

$$
\begin{aligned}
& \left(e^{2 \lambda}\right)_{(1,0)}=0=\left(e^{2 \beta}\right)_{(1,0)}, \\
& \left(e^{2 \gamma}\right)_{(1,0)}=0=\left(e^{2 \chi}\right)_{(1,0)}, \quad \omega_{(1,0)}=\frac{2 M a}{r^{3}} .
\end{aligned}
$$

To second order we find

$$
\begin{aligned}
& \left(e^{2 \lambda}\right)_{(2,0)}=\frac{4 M^{2} a^{2}}{r^{4}} \sin ^{4} \theta+\frac{2 M a^{2}}{r^{3}} \cos ^{2} \theta, \\
& \left(e^{2 \beta}\right)_{(2,0)}=\frac{a^{2}}{r^{2}}\left(1-\frac{2 M}{r}\right)^{-1}\left[\cos ^{2} \theta-\left(1-\frac{2 M}{r}\right)^{-1}\right] \text {, } \\
& \left(e^{2 \gamma}\right)_{(2,0)}=\frac{a^{2}}{r^{2}} \cos ^{2} \theta \text {, } \\
& \left(e^{2 \chi}\right)_{(2,0)}=\frac{a^{2}}{r^{2}}\left(1+\frac{2 M}{r}\right) \sin ^{2} \theta, \quad \omega_{(2,0)}=0,
\end{aligned}
$$

We model the stress-energy tensor of matter in the neutron star as that of a perfect fluid

$$
T_{a b}^{\mathrm{mat}}=(\rho+p) u_{a} u_{b}+p g_{a b},
$$

where $p \equiv p(r)$ and $\rho \equiv \rho(r)$ are the pressure and density, while $u^{a}=\left(u^{0}, 0,0, \Omega u^{0}\right)$ is the fluid's four-velocity that corresponds to a constant angular velocity $\Omega$ (we do not consider differentially rotating stars here). Normalization of this four-velocity requires $u_{a} u^{a}=-1$, and hence

$$
\begin{aligned}
u^{0} & =e^{-\lambda_{(0,0)}}\left[1-\epsilon^{2} \lambda_{(2,0)}-\epsilon \zeta \lambda_{(1,1)}\right. \\
& \left.+\frac{1}{2} \epsilon^{2} e^{-2 \lambda_{(0,0)}} r^{2} \sin ^{2} \theta\left(\Omega-\omega_{(1,0)}\right)^{2}\right] .
\end{aligned}
$$

We now expand the stress-energy tensor order by order in $\epsilon=R \Omega \ll 1$. To zeroth-order, the only non-vanishing 
components are

$$
\begin{aligned}
{ }^{(0,0)} T_{t t}^{\text {mat }} & =\rho e^{2 \lambda^{(0,0)}}, \quad{ }^{(0,0)} T_{r r}^{\text {mat }}=p e^{2 \beta^{(0,0)}} \\
{ }^{(0,0)} T_{\theta \theta}^{\text {mat }} & =p r^{2}=\frac{{ }^{(0,0)} T_{\phi \phi}^{\text {mat }}}{\sin ^{2} \theta}
\end{aligned}
$$

To first order in $\epsilon$, the non-vanishing terms are

$$
{ }^{(1,0)} T_{t \phi}^{\mathrm{mat}}=-r^{2} \sin ^{2} \theta\left[\rho\left(\Omega-\omega^{(1,0)}\right)+p \Omega\right],
$$

while to second order in $\epsilon$ we find

$$
\begin{aligned}
{ }^{(2,0)} T_{t t}^{\text {mat }} & =2 \lambda_{(2,0)} \rho e^{2 \lambda^{(0,0)}}+r^{2} \sin ^{2} \theta\left[\rho\left(\Omega^{2}-\omega_{(1,0)}^{2}\right)\right. \\
& \left.+p \Omega^{2}\right]+2 p r^{2} \sin ^{2} \theta\left(\gamma_{(2,0)}+\chi_{(2,0)}\right), \\
{ }^{(2,0)} T_{r r}^{\text {mat }} & =2 p e^{2 \beta^{(0,0)}} \beta_{(2,0)}, \\
{ }^{(2,0)} T_{\phi \phi}^{\text {mat }} & =e^{-2 \lambda_{(0,0)}} r^{4}\left(\omega^{(1,0)}-\Omega\right)^{2}(p+\rho) \sin ^{4} \theta .
\end{aligned}
$$

The cross terms of $\mathcal{O}(1,1)$ are

$$
\begin{aligned}
& { }^{{ }^{(1,1)}} T_{t t}^{\text {mat }}=2 \lambda_{(1,1)} \rho e^{2 \lambda^{(0,0)}}, \\
& { }^{{ }^{(1,1)}} T_{r r}^{\text {mat }}=2 p e^{2 \beta^{(0,0)}} \beta_{(1,1)}, \\
& { }^{{ }^{(1,1)}} T_{t \phi}^{\text {mat }}=r^{2} \rho \omega_{(1,1)} \sin ^{2} \theta . \\
& { }^{(1,1)} T_{\phi \phi}^{\text {mat }}=2 p r^{2} \sin ^{2} \theta\left(\gamma_{(1,1)}+\chi_{(1,1)}\right) .
\end{aligned}
$$

The trace is always $T^{\text {mat }} \equiv g^{a b} T_{a b}^{\text {mat }}=3 p-\rho$ up to $\mathcal{O}\left(\epsilon^{3}\right)$. Notice that the density and the pressure are assumed to be independent of $\Omega$ and $\alpha$, and thus they carry no $\epsilon$ or $\zeta$ dependence.

The components of the stress-energy obtained in this section depend only on the perfect-fluid assumption of Eq. (25) and the stationarity and axisymmetry assumption of the metric of Eq. (20). In general, for an arbitrary metric tensor, all components of the stress-energy will be non-vanishing, but here many components vanish due to the axisymmetry condition. Moreover, in the presence of electromagnetic fields, there will be additional contributions that are not accounted for here.

The EOS determines the relation between the density and the pressure: $p=p(\rho)$. We shall employ here the FPS EOS [90], which predicts neutron-star masses smaller than $2 M_{\odot}$ and radii ( $\simeq 10 \mathrm{~km}$; see Fig. 1) that are consistent with recent observations of X-ray bursters.

\section{NEUTRON STARS IN DYNAMICAL CS MODIFIED GRAVITY}

In this section, we solve the modified field equations in the small-coupling and slow-rotation approximation (within the perturbative constraint framework). Each subsection considers the equations at each successive order in the perturbation theory ladder.

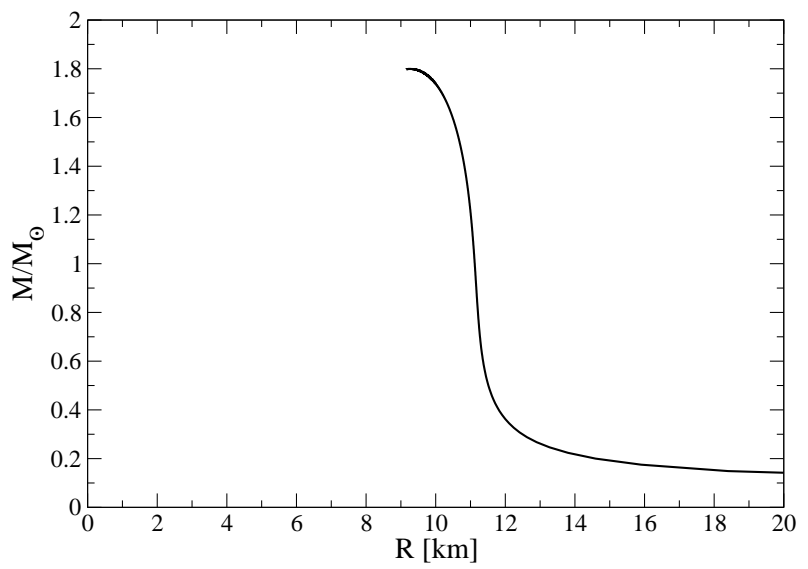

FIG. 1: The mass-radius relation for neutron stars calculated using the FPS EOS. Notice that for the FPS EOS, neutron stars are smaller than $2 M_{\odot}$ and have radii $\sim 10 \mathrm{~km}$.

\section{A. Zeroth-Order Field Equations}

We first study the field equations to $\mathcal{O}(0,0)$, i.e., the solutions that are independent of $\zeta$ and $\epsilon$. To this order, the C-tensor can be neglected, as it is at least linear in $\zeta$ and $\epsilon$. The field equations then reduce exactly to Einstein's equations (for a review of neutron star solution in GR see e.g. [23, 24, 91]). The Einstein tensor possesses only three independent components: $(t, t),(r, r)$ and $(\theta, \theta)$. Using the definition $m(r) \equiv r\left(1-e^{-2 \beta}\right) / 2$, the $(t, t)$ and the $(r, r)$ components reduce to the mass conservation equation and an equation for the $\lambda$ metric function, respectively

$$
\begin{aligned}
\frac{2 m_{(0,0)}^{\prime}}{r^{2}} & =8 \pi \rho, \\
\lambda_{(0,0)}^{\prime} & =\frac{m_{(0,0)}+4 \pi p r^{3}}{r\left(r-2 m_{(0,0)}\right)} .
\end{aligned}
$$

Hereafter, a prime stands for differentiation with respect to radius. We have explicitly included the index $(0,0)$ here to remind us that we are dealing with the $\mathcal{O}(0,0)$ coefficients of the metric expansion.

The system of differential equations becomes complete once we specify a third differential equation relating the rate of change of pressure to the density. We could use the $(\theta, \theta)$ component, but the equations simplify the most if we instead use the equations of stress-energy conservation (which hold to all orders in dynamical CS modified gravity): $\nabla^{\mu} T_{\mu \nu}^{\text {mat }}=0$. The $r$-component of this equation becomes

$$
\nabla^{\mu} T_{\mu r}^{\text {mat }}=\lambda_{(0,0)}^{\prime}(\rho+p)+p^{\prime}=0 .
$$

Combining this equation with Eq. (32) we obtain the Tolman-Oppenheimer-Volkoff (TOV) equation

$$
p^{\prime}=-\frac{m_{(0,0)}+4 \pi p r^{3}}{r\left(r-2 m_{(0,0)}\right)}(\rho+p) .
$$


Given an EOS relating density and pressure, we can then solve the system of equations (31)-(34).

\section{B. Zeroth-Order Scalar-Field Evolution Equation}

Once we have obtained the metric functions $\lambda_{(0,0)}$ and $\beta_{(0,0)}$, we solve the evolution equation for the CS scalar field to zeroth order in $\epsilon$ and $\zeta$. To this order, however, the spacetime is completely spherically symmetric. In 64], it was shown that for such spacetimes, the Pontryagin density identically vanishes, i.e., ${ }^{*} R R=0$, which then forces the evolution equation to become

$$
\square \vartheta^{(0,0)}=0 \text {. }
$$

Here again the $(0,0)$ index reminds us that we are working to zeroth-order in both perturbation parameters.

Since we are searching for a stationary and axisymmetric solution, $\vartheta$ can depend only on $(r, \theta)$. With this assumption, the above differential equation becomes

$$
\begin{aligned}
\square \vartheta_{(0,0)} & =e^{-2 \beta^{(0,0)}} \vartheta_{, r r}^{(0,0)}+\vartheta_{, r}^{(0,0)} e^{-2 \beta^{(0,0)}}\left(\lambda_{(0,0)}^{\prime}-\beta_{(0,0)}^{\prime}\right. \\
& \left.+\frac{2}{r}\right)+\frac{1}{r^{2}} \vartheta_{, \theta \theta}^{(0,0)}+\frac{\cot \theta}{r^{2}} \vartheta_{, \theta}^{(0,0)} .
\end{aligned}
$$

In fact, the above differential equation also holds to $\mathcal{O}(1,0)$ with the substitution $\vartheta^{(0,0)} \rightarrow \vartheta^{(1,0)}$.

This differential equation only possesses ill-behaved solutions, i.e., solutions that lead to a scalar field with infinite energy. Such an observation has in fact already been made when investigating the exterior gravitational field of slowly-rotating black holes [45]. The only solution that is consistent with a scalar field with finite energy is $\vartheta^{(0,0)}=$ const., which is the choice we make here. Of course, such a scalar field leads to no modification to the field equations as the $\mathrm{C}$-tensor depends on $\vartheta$ derivatives.

\section{First-Order Field Equations}

We now search for solutions of the modified field equations that are either linear in $\epsilon$ or linear in $\zeta$, i.e., solutions to $\mathcal{O}(1,0)$ or $\mathcal{O}(0,1)$. As already argued, however, there are no CS corrections independent of $\epsilon$, and thus $\omega_{(0,1)}=0$. As for corrections of $\mathcal{O}(1,0)$, the C-tensor does not contribute, while the Einstein tensor contains only one equation, the $(t, \phi)$ component, which reduces in normal form to

$$
\omega_{(1,0)}^{\prime \prime}+D \omega_{(1,0)}^{\prime}+V \omega_{(1,0)}=-16 \pi \Omega \frac{\rho+p}{1-2 m_{(0,0)} / r} .
$$

Here, the dissipative and potential term respectively are

$$
\begin{aligned}
D & \equiv \frac{4}{r}-\left(\lambda_{(0,0)}^{\prime}+\beta_{(0,0)}^{\prime}\right) \\
V & \equiv 2 \beta_{(0,0)}^{\prime} \lambda_{(0,0)}^{\prime}-\frac{2}{r}\left(\lambda_{(0,0)}^{\prime}-\beta_{(0,0)}^{\prime}\right) \\
& -2 \lambda_{(0,0)}^{\prime 2}-2 \lambda_{(0,0)}^{\prime \prime}-16 \pi e^{2 \beta_{(0,0)} \rho .}
\end{aligned}
$$

We can simplify the above expressions using the zeroth order ones in Eqs. (31)-(34). Doing so we find

$$
\begin{aligned}
D & =\frac{4}{r}-\frac{4 \pi r^{2}(\rho+p)}{r-2 m_{(0,0)}}, \\
V & =-\frac{16 \pi r}{r-2 m_{(0,0)}}(\rho+p) .
\end{aligned}
$$

We consider some analytic solutions to this equations in the Appendix. Once more, once $\lambda, \beta, p$ and $\rho$ are known as functions of radius (from the previous rung of the perturbative ladder), the above equations can be solved to find $\omega_{(1,0)}$ as a function of $r$.

\section{First-Order Scalar Field Evolution Equation}

We now consider the evolution equation for the scalar field to $\mathcal{O}(1,0)$ or $\mathcal{O}(0,1)$, i.e., we search for an evolution equation for this scalar field that is linear in $\epsilon$ or linear in $\zeta$. The left-hand side of the evolution equation is given by Eq. (36), as we showed in Sec. IVB. The right-hand side of the evolution equation depends on the Pontryagin density, which to linear order is:

$$
\begin{aligned}
{ }^{*} R R_{(1,0)} & =\frac{8}{r^{2}} \cos \theta e^{-3 \beta_{(0,0)}-\lambda_{(0,0)}}\left[1-e^{2 \beta_{(0,0)}}\right. \\
& +r\left(\beta_{(0,0)}^{\prime}-\lambda_{(0,0)}^{\prime}\right)+r^{2}\left(\lambda_{(0,0)}^{\prime 2}-\lambda_{(0,0)}^{\prime} \beta_{(0,0)}^{\prime}\right. \\
& \left.\left.+\lambda_{(0,0)}^{\prime \prime}\right)\right] \omega_{(1,0)}^{\prime}
\end{aligned}
$$

We see immediately that the Pontryagin density is of $\mathcal{O}(1,0)$ and there is no $\mathcal{O}(0,1)$ piece, which immediately implies we can neglect $\vartheta_{(0,1)}$.

The complicated source for the evolution equation of the scalar field is greatly simplified once we make use of the lower-order equations. Inserting Eqs. (31)-(34), the Pontryagin density simplifies to

$$
\begin{aligned}
{ }^{*} R R_{(1,0)} & =-\frac{48}{r^{3}} e^{-\lambda_{(0,0)}} \cos \theta\left(m_{(0,0)}-\frac{4 \pi}{3} r^{3} \rho\right) \\
& \times \sqrt{1-\frac{2 m_{(0,0)}}{r}} \omega_{(1,0)}^{\prime} .
\end{aligned}
$$

where $\omega_{(1,0)}^{\prime}$ is to be understood as a known function obtained after solving Eq. (37). For completeness, we present the full evolution equation for the scalar field [i.e., Eq. (9) to first order in $\epsilon$ ] below:

$$
\begin{gathered}
e^{-2 \beta_{(0,0)}} \vartheta_{, r r}^{(1,0)}+\vartheta_{, r}^{(1,0)} e^{-2 \beta_{(0,0)}}\left(\lambda_{(0,0)}^{\prime}-\beta_{(0,0)}^{\prime}+\frac{2}{r}\right) \\
+\frac{1}{r^{2}} \vartheta_{, \theta \theta}^{(1,0)}+\frac{\cot \theta}{r^{2}} \vartheta_{, \theta}^{(1,0)}=\frac{12 \alpha}{r^{3}} e^{-\lambda_{(0,0)} \cos \theta\left(m_{(0,0)}\right.} \\
\left.-\frac{4 \pi}{3} r^{3} \rho\right) \sqrt{1-\frac{2 m_{(0,0)}}{r}} \omega_{(1,0)}^{\prime}
\end{gathered}
$$




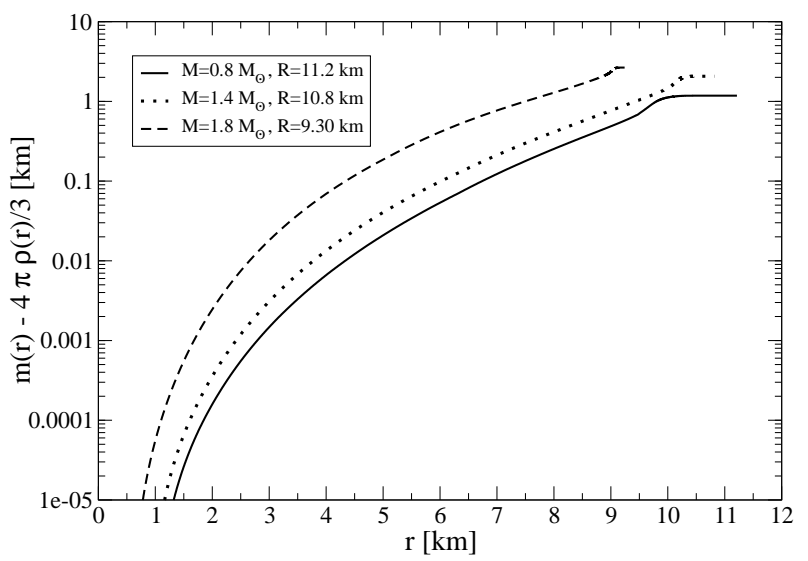

FIG. 2: The CS sourcing function $m_{(0,0)}-4 \pi r^{3} \rho / 3$ as a function of radius in $\mathrm{km}$. Close to the core, the function vanishes, while it reaches its maximum near the surface. The three curves correspond to stars with different central densities and hence with different masses.

Before proceeding with the solutions, it is worth studying the structure of the Pontryagin constraint. The quantity in between parenthesis in Eq. (43) is simply

$$
m_{(0,0)}-\frac{4 \pi}{3} r^{3} \rho=\frac{4 \pi}{3} r^{3}(\bar{\rho}-\rho),
$$

where we have defined the mean density $\bar{\rho} \equiv$ $3 m_{(0,0)} /\left(4 \pi r^{3}\right)$. Clearly, for constant density stars, the above correction identically vanishes and the leading order term of the Pontryagin density is of $\mathcal{O}\left(\epsilon^{3}\right)$. This is indeed the case for the EOS employed in 63], which would incorrectly suggest that for such an EOS there is no observable CS correction to neutron stars. Note here that there is no $\epsilon^{2}$ contribution to ${ }^{*} R R$ because a term of this order is parity invariant, while only odd-powers of $\epsilon$ can break this symmetry.

For non-constant density EOSs, one expects the above density-dependent quantity to be close to zero near the neutron star core and reach its maximum value close to its surface, a behavior that is verified in Fig. 2. This figure shows the relevant term as a function of radius in units of kilometers. The three curves correspond to three different central densities that lead to different total masses. Clearly, in the core (near zero radius), the Pontryagin density is nearly vanishing as the density is nearly constant, while it reaches its maximum near the surface of the star, which is close in magnitude to the total mass of the star. This behavior implies that the scalar field will be driven to non-vanishing values near the surface, which then in turn will lead to modifications to the gravitational field near the surface of the star. Due to this density dependence, the CS correction is not very sensitive to uncertainties in the nuclear physics, as the EOS near the neutron star surface is fairly robust.

A physical reason behind the vanishing of ${ }^{*} R R$ for constant-density stars is the following. In [64], it was shown that ${ }^{*} R R=E_{a b} B^{a b}$, where $E_{a b}$ and $B_{a b}$ are the electric and magnetic tidal tensors, which can be constructed from the Weyl tensor. Such a relation allows for an analogy between CS modified gravity and electromagnetic theory. In the latter, we know that the equations of motion for electromagnetic radiation satisfy wave equations in vacuum, but in the presence of matter, these equations acquire sources, e.g. $\square E \propto \nabla \rho+\dot{J}$ and $\square B \propto \nabla \times J$, where $\rho$ and $J$ are the charge and current densities respectively. Thus, in the presence of matter, the inhomogeneous solutions to the electric and magnetic fields need not be perpendicular to each other, leading to a non-vanishing $E \cdot B$, and, by analogy, a non-vanishing Pontryagin density. It is no surprise then that ${ }^{*} R R$ is proportional to derivatives of the matter density and the current, the latter of which in the gravitational sector is proportional to derivatives of the $\omega$ metric perturbation.

\section{E. Second-Order Field Equations}

The field equations to second order can be split into two sets: that of $\mathcal{O}(1,1)$ and that of $\mathcal{O}(2,0)$ (as argued before, there is no correction to the metric of $\mathcal{O}(0,2))$. The latter reduces simply to the second-order GR equations of structure, which we do not present here. This is a result of the C-tensor vanishing identically to $\mathcal{O}(2,0)$. The $\mathcal{O}(1,1)$ equations contain CS modifications, as the $\mathrm{C}$-tensor does not vanish in this case.

The only surviving term of the C-tensor is

$$
\begin{array}{r}
\left(\frac{\alpha}{\kappa} C_{t \phi}\right)^{(1,1)}=-4 \pi \sin \theta e^{\lambda_{(0,0)}}\left(1-\frac{2 m_{(0,0)}}{r}\right)^{1 / 2} \\
{\left[\left(\frac{\bar{\rho}-\rho}{r}+\frac{d \rho}{d r}\right) \partial_{\theta} \vartheta_{(1,0)}-(\bar{\rho}-\rho) \partial_{r \theta} \vartheta_{(1,0)}\right] .}
\end{array}
$$

Since all quantities in this equation have already been computed, the $\mathrm{C}$-tensor effectively acts as a new source for the field equations (a new effective matter term). The accompanying component of the Einstein tensor to this order is given by

$$
\begin{aligned}
G_{t \phi}^{(1,1)} & =\frac{r^{2}}{2} \sin ^{2} \theta\left(1-\frac{2 m_{(0,0)}}{r}\right) \partial_{r r} \omega_{(1,1)} \\
& +\sin ^{2} \theta\left[2 r\left(1-\frac{2 m_{(0,0)}}{r}\right)-2 \pi r^{3}(\rho+p)\right] \partial_{r} \omega_{(1,1)} \\
& -8 \pi p r^{2} \sin ^{2} \theta \omega_{(1,1)}+\frac{3}{2} \sin \theta \cos \theta \partial_{\theta} \omega_{(1,1)} \\
& +\frac{1}{2} \sin ^{2} \theta \partial_{\theta \theta} \omega_{(1,1)} .
\end{aligned}
$$

The stress-energy tensor to this order has already been given in a previous section. The full $(t, \phi)$ component of the modified field equations to $\mathcal{O}(1,1)$ is then 


$$
\begin{gathered}
\quad \frac{r^{2}}{2}\left(1-\frac{2 m_{(0,0)}}{r}\right) \partial_{r r} \omega_{(1,1)}+\left[2 r\left(1-\frac{2 m_{(0,0)}}{r}\right)-2 \pi r^{3}(\rho+p)\right] \partial_{r} \omega_{(1,1)}+\frac{1}{2}\left(3 \cot \theta \partial_{\theta} \omega_{(1,1)}+\partial_{\theta \theta} \omega_{(1,1)}\right) \\
-8 \pi(\rho+p) r^{2} \omega_{(1,1)}=4 \pi \frac{\alpha^{2}}{\kappa} \frac{e^{\lambda(0,0)}}{\sin \theta}\left(1-\frac{2 m_{(0,0)}}{r}\right)^{1 / 2}\left[\left(\frac{\bar{\rho}-\rho}{r}+\frac{d \rho}{d r}\right) \partial_{\theta} \vartheta_{(1,0)}-(\bar{\rho}-\rho) \partial_{r \theta} \vartheta_{(1,0)}\right]
\end{gathered}
$$

Given solutions for $\left(\lambda_{(0,0)}, m_{(0,0)}, \vartheta_{(1,0)}, p, \rho\right)$, we can now solve the above equation for the CS correction to the metric $\omega_{(1,1)}$.

We can analyze separately the other components of the field equations. First, we note that all other components of the C-tensor identically vanish to this order. Moreover, the only non-vanishing components of the stress-energy tensor are the $(t, t),(r, r)$ and $(\phi, \phi)$ ones, which are linearly proportional to $\lambda_{(1,1)}, \beta_{(1,1)}$ and $\left(\gamma_{(1,1)}, \chi_{(1,1)}\right)$ respectively. The Einstein tensor also possesses nonvanishing components that lead to differential equations for these metric functions. However, since the C-tensor vanishes and there are no metric-independent matter source terms at this order, we can set these metric perturbations to zero, i.e., $\left(\lambda_{(1,1)}, \beta_{(1,1)}, \chi_{(1,1)}, \gamma_{(1,1)}\right)=0$.

\section{F. Boundary Conditions}

The differential equations presented in the previous subsections must be complemented by boundary conditions. The exterior solution for the metric outside a slowly-rotating star in dynamical CS modified gravity was found in [45]. The line element was found to be

$$
\begin{aligned}
d s_{\mathrm{ext}}^{2} & =d s_{\mathrm{K}}^{2}+\frac{5}{4} \frac{\alpha^{2}}{\kappa} \frac{a}{r^{4}}\left(1+\frac{12}{7} \frac{M}{r}+\frac{27}{10} \frac{M^{2}}{r^{2}}\right) \sin ^{2} \theta d t d \phi \\
\vartheta^{\mathrm{ext}} & =\frac{5}{8} \alpha \frac{a}{M} \frac{\cos \theta}{r^{2}}\left(1+\frac{2 M}{r}+\frac{18 M^{2}}{5 r^{2}}\right)
\end{aligned}
$$

where $d s_{\mathrm{K}}^{2}$ is the slow-rotation limit of the Kerr line element. The solutions to the differential equations described in Sec. IVE must be guaranteed to approach the above solution in the limit $r \rightarrow R$, where $R$ is the neutron-star radius.

In terms of the metric functions, the CS correction only affects the $\omega_{(1,1)}$ term, which becomes, for the exterior metric:

$$
\omega_{(1,1)}^{\mathrm{ext}}=-\frac{5}{8} \frac{\alpha^{2}}{\kappa} \frac{a}{r^{6}}\left(1+\frac{12}{7} \frac{M}{r}+\frac{27}{10} \frac{M^{2}}{r^{2}}\right) .
$$

This relation is derived from the fact that $g_{t \phi}^{(1,0)}=$ $-r^{2} \sin ^{2} \theta \omega_{(1,0)}$. The metric components corresponding to the Kerr metric have already been given in Eqs. (22)(24). The boundary conditions are then simply applied by requiring that all metric functions approach their exterior modified Kerr counterparts at $r=R$.

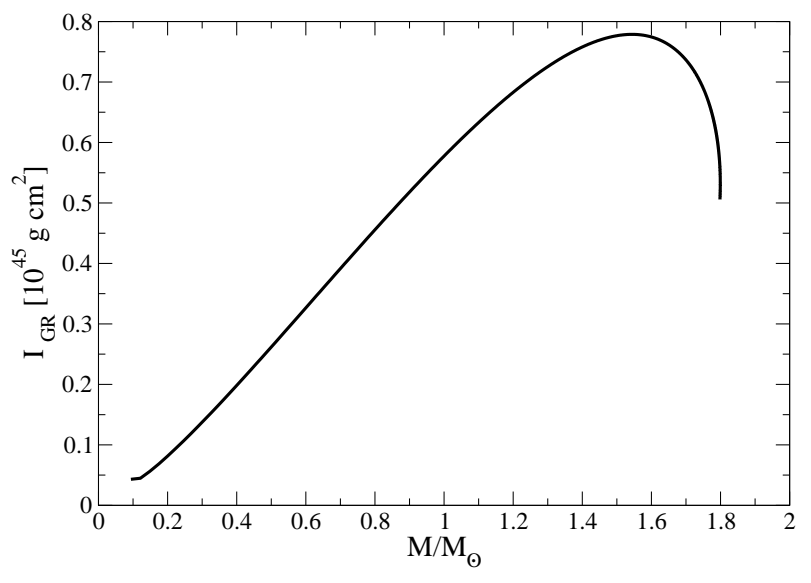

FIG. 3: The moment of inertia of neutron stars described by the FPS EOS, as a function of their masses.

\section{NUMERICAL SOLUTIONS}

In this section we present results for the numerical solution to the modified field equations. We begin with the standard GR functions and proceed to solutions for the CS scalar field and the CS correction to the metric.

\section{A. General Relativistic Solutions}

We first solve numerically the GR equations of neutron star structure for the FPS EOS. We use standard numerical techniques to solve these equations: we reduce the system of equations (31), (32), (34) and (37) to first order and then employ a fourth-order, Runge-Kutta scheme to obtain a numerical solution. The boundary conditions for the GR solutions are implemented following the scheme of [89, 92], which exploits the scale-free nature of the differential equations.

We have already shown the mass-radius relation in Fig. 1. From the $g_{t \phi}$ metric component, we now compute the moment of inertia via

$$
I \equiv \frac{8 \pi}{3} \int_{0}^{R} r^{4}(\rho+p) \sqrt{\frac{g_{r r}}{g_{t t}}}\left(1-\frac{\omega(r, \theta)}{\Omega}\right) d r .
$$

Here $g_{t t}$ and $g_{r r}$ are the $(t, t)$ and $(r, r)$ components of the metric. We plot this quantity in Fig. 3 as a function of neutron-star mass. 


\section{B. First-Order CS Scalar Field Solution}

In order to solve Eq. (44), we will first convert this partial differential equation to an ordinary one via separation of variables, i.e.,

$$
\vartheta^{(1,0)}(r, \theta)=\sum_{n} \bar{\vartheta}_{n}(r) P_{n}(\cos \theta)
$$

where $P_{n}$ are the Legendre polynomials 62] and we have omitted the order symbols in $\bar{\vartheta}$ for convenience.

The $\theta$-dependent term in Eq. (44) is nothing but

$$
\vartheta_{, \theta \theta}^{(1,0)}+\cot \theta \vartheta_{, \theta}^{(1,0)}=\frac{1}{\sin \theta} \partial_{\theta}\left(\sin \theta \vartheta_{, \theta}^{(1,0)}\right)
$$

When we evaluate this with respect to Legendre polynomials, we find

$$
\frac{1}{\sin \theta} \partial_{\theta}\left(\sin \theta P_{n, \theta}\right)=-n(n+1) P_{n}(\cos \theta)
$$

using the master Legendre polynomial equation. With this at hand, Eq. (44) becomes

$$
\begin{aligned}
e^{-2 \beta_{(0,0)}} \bar{\vartheta}_{n}^{\prime \prime}+ & \bar{\vartheta}_{n}^{\prime} e^{-2 \beta_{(0,0)}}\left(\lambda_{(0,0)}^{\prime}-\beta_{(0,0)}^{\prime}+\frac{2}{r}\right) \\
-\frac{n(n+1)}{r^{2}} \bar{\vartheta}_{n}= & \frac{12 \alpha}{r^{3}} e^{-\lambda_{(0,0)}} \frac{\cos \theta}{P_{n}}\left(m_{(0,0)}\right. \\
& \left.-\frac{4 \pi}{3} r^{3} \rho\right) \sqrt{1-\frac{2 m_{(0,0)}}{r}} \omega_{(1,0)}^{\prime} .
\end{aligned}
$$

Clearly, all $\theta$ dependence disappears once we choose $n=1$, such that $P_{1}=\cos \theta$ and $\vartheta^{(1,0)}=\bar{\vartheta} \cos \theta$. This ordinary differential equation becomes

$$
\begin{aligned}
& e^{-2 \beta_{(0,0)}} \bar{\vartheta}_{1}^{\prime \prime}+\bar{\vartheta}_{1}^{\prime} e^{-2 \beta_{(0,0)}}\left(\lambda_{(0,0)}^{\prime}-\beta_{(0,0)}^{\prime}+\frac{2}{r}\right)-\frac{2}{r^{2}} \bar{\vartheta}_{1} \\
& =\frac{12 \alpha}{r^{3}} e^{-\lambda_{(0,0)}}\left(m_{(0,0)}-\frac{4 \pi}{3} r^{3} \rho\right) \sqrt{1-\frac{2 m_{(0,0)}}{r}} \omega_{(1,0)}^{\prime},
\end{aligned}
$$

which we can simplify even more using the lower-order TOV equations. The result is

$\bar{\vartheta}^{\prime \prime}+\bar{\vartheta}^{\prime}\left(1-\frac{2 m_{(0,0)}}{r}\right)^{-1}\left[4 \pi r(p-\rho)+\frac{2}{r}\left(1-\frac{m_{(0,0)}}{r}\right)\right]$

$-\frac{2 \bar{\vartheta}}{r^{2}}\left(1-\frac{2 m_{(0,0)}}{r}\right)^{-1}=\frac{12 \alpha}{r^{3}} e^{-\lambda_{(0,0)}}\left(m_{(0,0)}-\frac{4 \pi}{3} r^{3} \rho\right)$

$\times\left(1-\frac{2 m_{(0,0)}}{r}\right)^{-1 / 2} \omega_{(1,0)}^{\prime}$.

Equation (56) is an ordinary differential equation (of dipole, $n=1$, type) that must be solved for $\bar{\vartheta}_{1}$. The boundary conditions for this function are the following: at the neutron star surface, $\bar{\vartheta}_{1} \cos \theta$ should equal Eq. (49); at the center of the core, $\bar{\vartheta}_{1}^{\prime}=0$, as there can be no CS

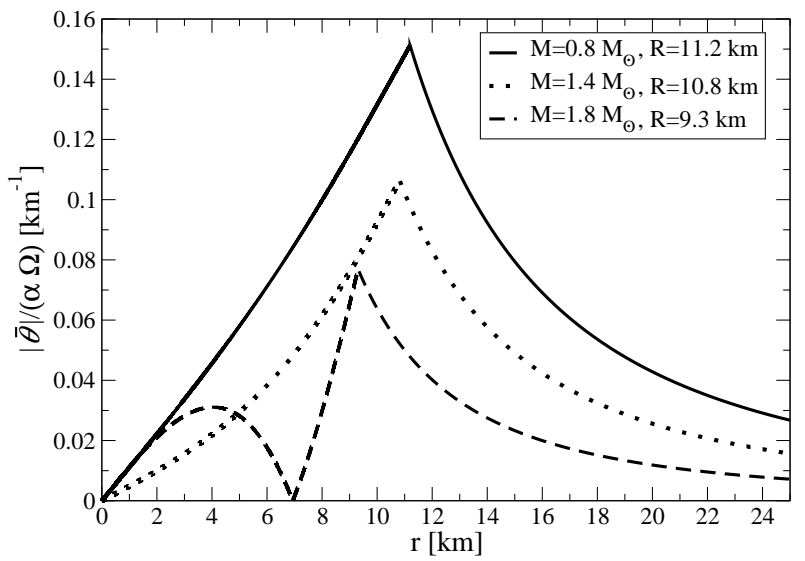

FIG. 4: The absolute value of the dipolar component of the scalar field $\bar{\vartheta}$ as a function of radius, for three stars with different masses and radii.

correction when the spin angular momentum vanishes. This, of course, presents a numerical challenge, as the above differential equation is not scale free. We choose to use a shooting method, where we iterate over a variety of boundary conditions at the core for $\bar{\vartheta}_{1}$ until the surface boundary condition is satisfied.

Figure 4 shows the absolute value of the CS scalar field in units of $\mathrm{km}^{-1}$ as a function of radius. In this plot, we have divided $\bar{\vartheta}$ by $\alpha \Omega$ to give it units of inverse kilometers, since $\alpha$ has units of squared length and $\Omega$ of inverse length. The interior solution $(r<R)$ is obtained by numerically solving Eq. (57). The exterior solution $(r>R)$ is simply that of Eq. (49). Note that the CS scalar overall increases in magnitude until the surface of the star, at which points it decays as the radius cubed. This occurs because as the radius increases toward the surface, the spin angular momentum increases until it saturates at $r=R$. It is precisely this quantity (the spin angular momentum) that the CS scalar field couples to.

The behavior of $\bar{\vartheta}$ is slightly different for the most massive star with $M=1.8 M_{\odot}$ relative to the less massive ones. The difference in behavior is rooted in the source term of the evolution equation for the scalar field [the right-hand side of Eq. (57)]. This source is proportional to $\omega_{(1,0)}^{\prime}$, which by definition is proportional to $d I_{\mathrm{GR}} / d r$. Figure 3 shows that $d I_{\mathrm{GR}} / d r$ changes sign (ie. the slope of the curve changes sign) at around $M=1.4 M_{\odot}: d I_{\mathrm{GR}} / d r>0$ for $M<1.4 M_{\odot}$, while $d I_{\mathrm{GR}} / d r<0$ for $M>1.4 M_{\odot}$. In turn, this implies that the source term in Eq. (57) also changes sign. The change in sign of the source term forces $\bar{\vartheta}$ to start negative near the core, but quickly this quantity must switch sign so that the boundary condition in Eq. (49) is satisfied at the neutron star surface. This then explains why $\bar{\theta}$ behaves differently inside the neutron star for stellar configurations with masses larger or smaller than $1.4 M_{\odot}$. 


\section{Solution to the Second-Order Field Equations}

As before, we solve Eq. (48) numerically. However, it is again convenient to convert this partial differential equation into an ordinary one via separation of variables, i.e.,

$$
\omega_{(1,1)}(r, \theta)=\sum_{n} \bar{\omega}_{n}(r)\left[\frac{1}{\sin \theta} \partial_{\theta} P_{n}(\cos \theta)\right] .
$$

Such a functional dependence for the angular sector is chosen so that the left-hand side of Eq. (48) simplifies to

$$
\begin{gathered}
3 \cot \theta \partial_{\theta}\left(\frac{\partial_{\theta} P_{n}}{\sin \theta}\right)+\partial_{\theta \theta}\left(\frac{\partial_{\theta} P_{n}}{\sin \theta}\right)= \\
-(n+2)(n-1)\left(\frac{\partial_{\theta} P_{n}}{\sin \theta}\right)
\end{gathered}
$$

after repeated application of Eq. (54). Using this relation and inserting Eq. (52), Eq. (48) becomes

$$
\begin{gathered}
\frac{r^{2}}{2}\left(1-\frac{2 m_{(0,0)}}{r}\right) \bar{\omega}_{n}^{\prime \prime}\left(\frac{\partial_{\theta} P_{n}}{\sin \theta}\right)+\left[2 r\left(1-\frac{2 m_{(0,0)}}{r}\right)-2 \pi r^{3}(\rho+p)\right] \bar{\omega}_{n}^{\prime}\left(\frac{\partial_{\theta} P_{n}}{\sin \theta}\right)-\frac{\bar{\omega}_{n}}{2}(n+2)(n-1)\left(\frac{\partial_{\theta} P_{n}}{\sin \theta}\right) \\
-8 \pi(\rho+p) r^{2} \bar{\omega}_{n}\left(\frac{\partial_{\theta} P_{n}}{\sin \theta}\right)=-4 \pi \frac{\alpha^{2}}{\kappa} e^{\lambda_{(0,0)}}\left(1-\frac{2 m_{(0,0)}}{r}\right)^{1 / 2}\left[\left(\frac{\bar{\rho}-\rho}{r}+\frac{d \rho}{d r}\right) \bar{\vartheta}_{1}-(\bar{\rho}-\rho) \bar{\vartheta}_{1}^{\prime}\right]
\end{gathered}
$$

where, in the right-hand side, only the $n=1$ term survives in the $\vartheta$ expansion. Once more, for the lefthand side of this equation to be $\theta$ independent, we must choose $n=1$ in the $\omega^{(1,1)}$ expansion, which then ren$\operatorname{ders} \omega^{(1,1)}=-\bar{\omega}_{1}$. The above differential equation then simplifies to

$$
\begin{aligned}
& \frac{r^{2}}{2}\left(1-\frac{2 m_{(0,0)}}{r}\right) \bar{\omega}_{1}^{\prime \prime}+\left[2 r\left(1-\frac{2 m_{(0,0)}}{r}\right)\right. \\
- & \left.2 \pi r^{3}(\rho+p)\right] \bar{\omega}_{1}^{\prime}-8 \pi(\rho+p) r^{2} \bar{\omega}_{1}=4 \pi \frac{\alpha^{2}}{\kappa} e^{\lambda_{(0,0)}} \\
\times & \left(1-\frac{2 m_{(0,0)}}{r}\right)^{1 / 2}\left[\left(\frac{\bar{\rho}-\rho}{r}+\frac{d \rho}{d r}\right) \bar{\vartheta}_{1}-(\bar{\rho}-\rho) \bar{\vartheta}_{1}^{\prime}\right] .
\end{aligned}
$$

Given that $\bar{\vartheta}_{1}$ is known, the above equation is simply an ordinary differential equation for $\bar{\omega}_{1}$.

The boundary conditions for Eq. (61) are the following: at the neutron star surface, $\bar{\omega}_{1}$ is given by Eq. (50); at the center of the core, $\bar{\omega}_{1}^{\prime}=0$, as, once more, there can be no CS correction when the spin vanishes. As before, we employ a shooting method, where we iterate over boundary conditions at the core for $\bar{\omega}_{1}$ until the surface boundary condition is satisfied.

In Fig. 5 we plot the absolute value of the CS correction to the gravitomagnetic metric component in units of $\mathrm{km}^{-4}$ as a function of radius for three stars with different masses and radii. The interior solution presents a rather constant behavior for the two less massive stars, while the exterior solution always decays with the sixth power of the radius, as expected.

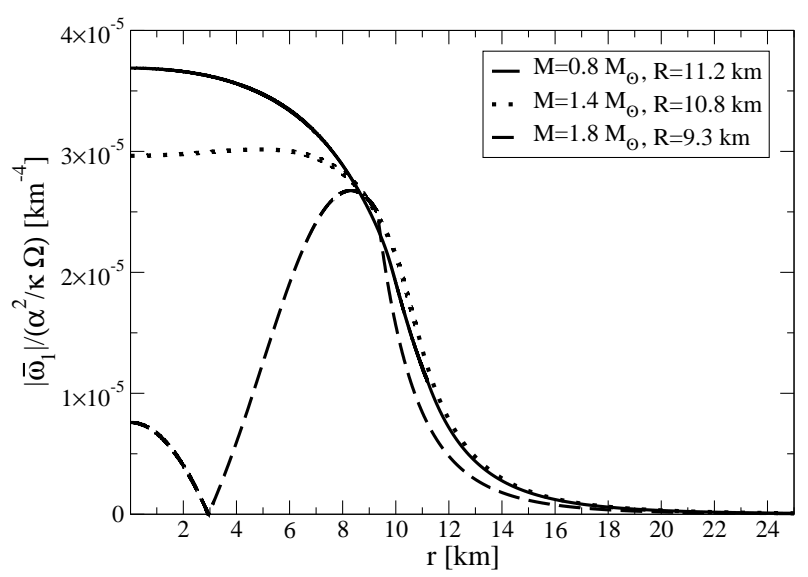

FIG. 5: The absolute value of the CS correction to the metric coefficient $\bar{\omega}_{1}$ as a function of radius inside and outside a neutron star, for stars with three different masses and radii.

\section{CONNECTION TO OBSERVABLES}

In this section, we connect the above CS modifications to observable quantities, in order to investigate whether neutron star observations can constrain the CS coupling parameter. For simplicity, let us define $\xi \equiv \alpha^{2} / \kappa$, such that $\zeta=\xi / R^{4}$. A constraint on the CS coupling $\alpha$ is thus a constraint on $\xi$. It is customary to quote constraints on $\xi^{1 / 4} \propto \alpha^{1 / 2}$, as this quantity has units of length.

The mass continuity equation and the equation of hydrostatic equilibrium are CS modified to subleading order, i.e., $\mathcal{O}(2,1)$, because the $\omega$ expansion (see Eq. (21)) cannot have a $\omega_{(0,1)}$ piece and any correction to the equation of hydrostatic equilibrium is proportional to $\omega^{2} \sim \omega_{(1,0)}^{2}+2 \omega_{(1,0)} \omega_{(1,1)}+2 \omega_{(1,0)} \omega_{(2,0)}$. This implies 
that CS modifications to the mass-radius relation scale as $\omega_{(1,0)} \omega_{(1,1)} \sim\left(\xi / R^{4}\right)(M / R)(R \Omega)^{2}$, which is clearly suppressed by a factor of $(R \Omega)^{2} \ll 1$, where the factor of $R^{2}$ must be present for dimensional consistency ${ }^{2}$. If the mass radius relation where measured to an accuracy of $\delta_{\mathrm{M}-\mathrm{R}} \sim 10 \%$, we could then place a constraint of order

$$
\begin{aligned}
\xi_{M-R}^{1 / 4} & \lesssim 55 \mathrm{~km}\left(\frac{R}{10 \mathrm{~km}}\right)\left(\frac{0.025}{R \Omega / c}\right)^{1 / 2} \\
& \times\left(\frac{\delta_{\mathrm{M}-\mathrm{R}}}{0.1}\right)^{1 / 4}\left(\frac{R}{10 \mathrm{~km}} \frac{1.6 M_{\odot}}{M}\right)^{1 / 4}
\end{aligned}
$$

which derives simply from requiring that $\delta_{\mathrm{M}-\mathrm{R}} \lesssim$ $\omega_{(1,1)} \omega_{(1,0)} /(M / R) \sim\left(\xi / R^{4}\right)(R \Omega)^{2}$. We have scaled the previous expression by $R=10 \mathrm{~km}, M=1.6 M_{\odot}$ and $\Omega /(2 \pi)=600 \mathrm{~Hz}$, corresponding to the typical radius and mass and the largest spin frequency of neutron stars bursters for which we have measurements [25]. Of course, for more slowly rotating pulsars, for example if $\Omega \sim 45 \mathrm{~Hz}$ $(\Omega \sim 0.36 \mathrm{~Hz})$, corresponding to the $\mathrm{A}(\mathrm{B})$ component of the double binary pulsar [79], then the bound worsens dramatically: $\xi_{M-R}^{1 / 4} \lesssim 220 \mathrm{~km}\left(\xi_{M-R}^{1 / 4} \lesssim 2400 \mathrm{~km}\right)$.

The weakness of the CS correction to the mass-radius relation of slowly-rotating stars is in fact an advantage. For sufficiently slowly-rotating stars, for example, for stars whose spin frequency is $\Omega \ll 10^{3} \mathrm{~Hz}$, corrections of $\mathcal{O}\left(\epsilon^{2}\right)$ to the mass-radius relation can be ignored. In such cases, the degeneracy between EOS and CS corrections is broken. That is, given a measurement of the mass-radius relation for a sub-kHz neutron star, the CS correction can be ignored, and the mass-radius relation can be used to infer the EOS.

A stronger constraint arises from the measurement of the moment of inertia of a neutron star, as is suggested by the modification to the gravitomagnetic sector of the metric [see Eq. (51)]. Since the metric function $\omega$ is CS modified, we infer that $I$ is also modified. We then decompose $I=I_{\mathrm{GR}}+I_{\mathrm{CS}}$, where the latter is given by Eq. (51) with the substitution $(1-\omega / \Omega) \rightarrow-\omega_{(1,1)} / \Omega$ and $\left(g_{t t}, g_{r r}\right)$ given by their GR values. Since the moment of inertia scales by definition as $1 / \Omega$, the CS modification is first-order in $\epsilon$. We can then infer that roughly $I \sim I_{\mathrm{GR}}\left(1+\xi / R^{4}\right)$, and assuming that $I$ has been measured to an accuracy $\delta_{I}$ (and found to agree with the GR result), we could infer the following constraint on $\xi$ :

$$
\xi_{I}^{1 / 4} \lesssim 5 \mathrm{~km}\left(\frac{R}{10 \mathrm{~km}}\right)\left(\frac{\delta_{I}}{0.1}\right)^{1 / 4}
$$

Here we have assumed again a fiducial neutron-star radius of $10 \mathrm{~km}$ and the nominal accuracy for the error in the measurement of the moment of inertia of $10 \%$ [7, 79].

\footnotetext{
2 This relation can also be obtained by substituting back into $\omega_{(1,0)} \omega_{(1,1)}$ the surface values required by the boundary conditions.
}

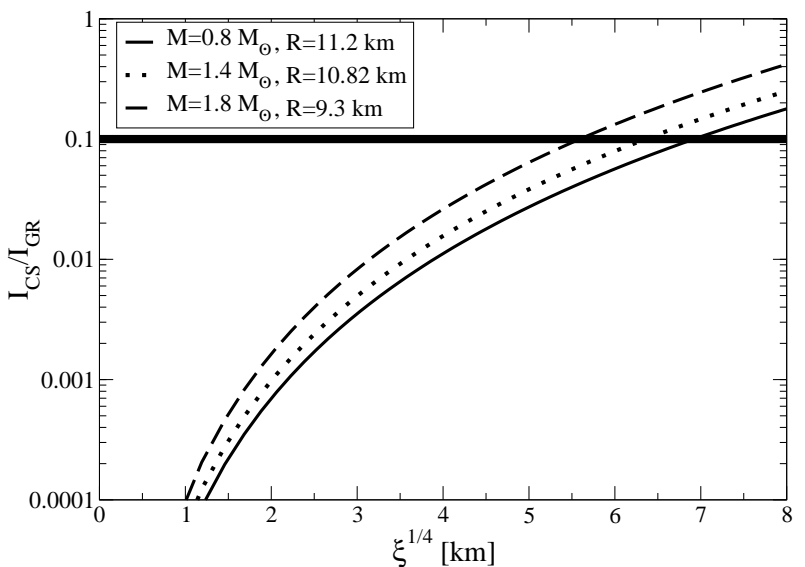

FIG. 6: The ratio between the CS correction to the moment of inertia and the GR value as a function of the CS coupling parameter $\xi^{1 / 4}$ in units of kilometers. The thick horizontal line corresponds to a measurement of the moment of inertia with an accuracy of $10 \%$.

As expected, this constraint is independent on the rotation rate of the neutron star.

Another way to estimate the magnitude of the constraint is to consider directly the $\omega$ metric perturbation. Since the moment of inertia depends linearly on $\omega$, a measurement of $I$ to an accuracy $\delta_{I}$ is effectively a measurement of $\omega$ to the same accuracy. Given then a measurement $\bar{\omega}$, we can infer $\bar{\omega}(1+\delta)=\omega_{(1,0)}\left(1+\omega_{(1,1)} / \omega_{(1,0)}\right)$, which leads to the constraint $\delta \lesssim \omega_{(1,1)} / \omega_{(1,0)}$, or simply

$$
\xi_{\omega}^{1 / 4} \lesssim 5 \mathrm{~km}\left(\frac{R}{10 \mathrm{~km}}\right)^{3 / 4}\left(\frac{M}{1.4 M_{\odot}}\right)^{1 / 4}\left(\frac{\delta_{I}}{0.1}\right)^{1 / 4}
$$

where we have here used Eqs. (23) and (50) to model the mass and radius dependence. As expected, this constraint is consistent with the one shown in Eq. (63).

In order to obtain more specific constraints on the CS coupling, we solved for the CS correction to the moment of inertia numerically. In Fig. 6] we plot the ratio of the CS correction to the moment of inertia to the GR value as a function of $\xi^{1 / 4}$ for three stars with different masses and radii (the lowest-mass case is shown for illustrative reasons, as no astrophysical process is known that leads to such light neutron stars). We remind the reader that we have previously defined $\xi \equiv \alpha^{2} / \kappa$. A $10 \%$ measurement implies a constraint of roughly $\xi^{1 / 4} \lesssim(5.5,6.5,7) \mathrm{km}$ for stars with final mass $M=(0.8,1.4,1.8) M_{\odot}$. These constraints are of the same order of magnitude as the rough estimates presented above. Also notice that the expansion parameter $\zeta=\xi / R^{4}$ is of order $\mathcal{O}\left(10^{-1}\right)$ if we saturate these constraints, which justifies the small-coupling approximation. We should emphasize that, unlike constraints derivable from measurements of the mass-radius relation, these constraints are independent of the spin frequency of the neutron star.

The possible bounds that one could achieve with moment of inertia measurements is to be contrasted from 
that obtained from observations of the orbital evolution of the double binary pulsar $\xi_{\text {binary }}^{1 / 4} \lesssim 10^{4} \mathrm{~km}[45]$. The moment-of-inertia bound will be much stronger because, in the binary pulsar case, the CS coupling parameter couples to the binary separation (roughly $10^{5} \mathrm{~km}$ ), which is much larger than the neutron star radius.

\section{CONCLUSIONS}

We have studied the structure of neutron stars in dynamical CS gravity. We have found that to leading order, only the gravitomagnetic sector of the metric is CS corrected. This implies that the mass-radius relation is only modified to second order, while the moment of inertia is modified to leading order. We have derived the CS modified equations of stellar structure and we have solved them numerically. These solutions complete the prescription of the gravitational field inside and outside of stars. We estimate that a measurement of the moment of inertia to $10 \%$ can lead to a constraint on the theory that is at least three orders of magnitude stronger than previous constraints.

As is typical with tests of alternative theories with neutron star observations, the observables are degenerate with the EOS, which leads to a degeneracy between alternative theory modifications and our knowledge of nuclear physics. In the case of CS gravity, however, the mass-radius relation can be used to extract the EOS, as neither the mass nor the radius are CS corrected to leading order. This is simply a consequence of the fact that CS gravity couples to leading-order to the spin angular momentum and not to the mass density.

Future observations that lead to a measurement of the moment of inertia of a neutron star would make the test presented here viable. The resulting constraint holds the promise of being one of the strongest achievable with any type of astrophysical observation, at least in the static sector of the theory. This analysis could then be complemented by gravitational wave tests, which sample CS corrections in the radiative sector.

\section{Acknowledgments}

We are grateful to Ramesh Narayan for his insights and assistance with the numerical solutions, as well as to David Spergel, Frans Pretorius and Branson Stephens for useful suggestions and comments. Some calculations used the computer algebra systems MAPLE, in combination with the GRTensorII package 93]. NY acknowledges the support of the NSF grant PHY-0745779. DP acknowledges the support of the NSF CAREER award PHY-0746549. This work was supported in part by NSF grant AST-0708640 for FÖ and NSF grant AST-0907890 for AL.

\section{Appendix A: Some Analytic Solutions to the Gravitomagnetic Sector of the Metric}

Equation (37) governs the behavior of the gravitomagnetic metric perturbation $\omega_{(1,0)}$. Unfortunately, there is no known closed-form, analytic solution for any realistic EOS. However, for some particularly simple EOSs, such a solution can in fact be found.

One example is to set $p=-\rho$, corresponding to a gravastar configuration [94, 95], i.e., a gravitational vacuum star, whose interior solution is diffeomorphic to De Sitter spacetime. In this case, the dissipative function becomes $D=4 / r$, the potential $V=0$, and the righthand side of Eq. (37) vanishes. We can then solve for the gravitomagnetic potential to find

$$
\omega_{(1,0)}=c_{1}+\frac{c_{2}}{r^{3}},
$$

where $c_{1,2}$ are two constants of integration. Finally, we can also solve the equation of hydrostatic equilibrium to obtain $p=c_{3}$, where $c_{3}$ is a constant of integration.

Perhaps a more realistic configuration is one with vanishing pressure and constant density $\rho=\rho_{\mathrm{c}}$. In this case, Ref. [63] has found that the GR solution is

$$
g_{t \phi}^{(1,0)}=\frac{4 \pi}{3} \rho_{\mathrm{c}} R^{2}(\vec{r} \times \vec{\Omega})_{\phi}\left[2-\frac{6}{5}\left(\frac{r}{R}\right)^{2}\right]
$$

assuming the fluid is rigidly rotating with angular velocity $\Omega$.

A third alternative is to allow $\rho+p=(r-$ $\left.2 m_{(0,0)}\right) /\left(\pi r^{3}\right)$. In this case, $D=0$ and $V=-16 / r^{2}$, while the right-hand side of Eq. (37) becomes equal to $-16 \Omega / r^{2}$. The gravitomagnetic component becomes

$$
\omega_{(1,0)}=\Omega+c_{1} r^{p_{1}}+c_{2} r^{p_{2}},
$$

where $p_{1,2}=1 / 2 \pm \sqrt{65}$ and $c_{1,2}$ are constant of integration.
[1] K. Nordtvedt, Phys. Rev. 169, 1017 (1968).

[2] C. M. Will and K. J. Nordtvedt, Astrophys. J. 177, 757 (1972).

[3] C. M. Will, Astrophys. J. 163, 611 (1971).

[4] K. J. Nordtvedt and C. M. Will, Astrophys. J. 177, 775 (1972).
[5] C. M. Will, Astrophys. J. 185, 31 (1973).

[6] C. M. Will, Living Reviews in Relativity 9 (2006), URL http://www. livingreviews.org/lrr-2006-3

[7] T. Damour, in Proceedings of the 2nd Canadian Conference on General Relativity and Relativistic Astrophysics (1988), pp. 315-334. 
[8] T. Damour and J. H. Taylor, Phys. Rev. D 45, 1840 (1992).

[9] M. Tegmark, Phys. Rev. D 66, 103507 (2002), arXiv:astro-ph/0101354.

[10] E. V. Linder and R. N. Cahn, Astropart. Phys. 28, 481 (2007), astro-ph/0701317.

[11] W. Hu and I. Sawicki, Phys. Rev. D76, 104043 (2007), 0708.1190.

[12] W. Fang, W. Hu, and A. Lewis, Phys. Rev. D78, 087303 (2008), 0808.3125.

[13] N. Yunes and F. Pretorius, Phys. Rev. D80, 122003 (2009), 0909.3328.

[14] F. D. Ryan, Phys. Rev. D56, 1845 (1997).

[15] N. A. Collins and S. A. Hughes, Phys. Rev. D69, 124022 (2004), gr-qc/0402063.

[16] K. Glampedakis and S. Babak, Class. Quant. Grav. 23, 4167 (2006), gr-qc/0510057.

[17] S. J. Vigeland and S. A. Hughes (2009), 0911.1756.

[18] D. Psaltis, Phys. Rev. D77, 064006 (2008), 0704.2426.

[19] D. Psaltis, Living Reviews in Relativity 11 (2008), URL http://www .livingreviews.org/lrr-2008-9.

[20] T. Damour (1982), based on set of four lectures given at Les Houches School on Gravitational Radiation, Les Houches, France, Jun 2-21, 1982.

[21] K. S. Thorne and J. B. Hartle, Phys. Rev. D31, 1815 (1984).

[22] R. H. Price and K. S. Thorne, Scientific American 258, 69 (1988).

[23] N. Glendenning, Compact Stars. Nuclear Physics, Particle Physics and General Relativity (Springer, 1996).

[24] J. M. Lattimer and M. Prakash, Astrophys. J. 550, 426 (2001), astro-ph/0002232.

[25] F. Ozel, T. Guver, and D. Psaltis, Astrophys. J. 693, 1775 (2009), 0810.1521.

[26] F. Ozel and D. Psaltis, Phys. Rev. D80, 103003 (2009), 0905.1959.

[27] T. P. Sotiriou and V. Faraoni (2008), 0805.1726.

[28] S. M. Carroll, V. Duvvuri, M. Trodden, and M. S. Turner, Phys. Rev. D70, 043528 (2004), astro-ph/0306438.

[29] T. Chiba, Phys. Lett. B575, 1 (2003), astro-ph/0307338.

[30] T. P. Sotiriou, Class. Quant. Grav. 23, 5117 (2006), grqc/0604028.

[31] G. R. Dvali, G. Gabadadze, and M. Porrati, Phys. Lett. B485, 208 (2000), hep-th/0005016.

[32] P. Horava, Phys. Rev. D79, 084008 (2009), 0901.3775.

[33] H. Lu, J. Mei, and C. N. Pope, Phys. Rev. Lett. 103, 091301 (2009), 0904.1595.

[34] J. D. Bekenstein, Phys. Rev. D70, 083509 (2004), astroph/0403694.

[35] J. D. Bekenstein and R. H. Sanders (2005), astro$\mathrm{ph} / 0509519$.

[36] S. Alexander and N. Yunes, Phys. Rept. 480, 1 (2009), 0907.2562.

[37] F. Moura and R. Schiappa, Classical and Quantum Gravity 24, 361 (2007), arXiv:hep-th/0605001.

[38] T. Damour and G. Esposito-Farese, Class. Quant. Grav. 9, 2093 (1992).

[39] T. Damour and G. Esposito-Farese, Phys. Rev. Lett. 70, 2220 (1993).

[40] T. Damour and G. Esposito-Farese, Phys. Rev. D54, 1474 (1996), gr-qc/9602056.

[41] A. Upadhye and W. Hu, Phys. Rev. D80, 064002 (2009), 0905.4055.

[42] E. Babichev and D. Langlois (2009), 0904.1382.
[43] E. Babichev and D. Langlois (2009), 0911.1297.

[44] A. Cooney, S. DeDeo, and D. Psaltis (2009), 0910.5480.

[45] N. Yunes and F. Pretorius, Phys. Rev. D79, 084043 (2009), 0902.4669.

[46] J. Polchinski (1998), vol. 2: Superstring theory and beyond.

[47] S. J. Gates, Jr., S. V. Ketov, and N. Yunes, Phys. Rev. D80, 065003 (2009), 0906.4978.

[48] S. H. S. Alexander and J. Gates, S. James, JCAP 0606, 018 (2006), hep-th/0409014.

[49] V. Taveras and N. Yunes, Phys. Rev. D78, 064070 (2008), 0807.2652.

[50] G. Calcagni and S. Mercuri, Phys. Rev. D79, 084004 (2009), 0902.0957.

[51] S. Mercuri and V. Taveras (2009), 0903.4407.

[52] S. Weinberg, Phys. Rev. D77, 123541 (2008), 0804.4291.

[53] R. Jackiw and S. Y. Pi, Phys. Rev. D68, 104012 (2003), gr-qc/0308071.

[54] S. Alexander and J. Martin, Phys. Rev. D71, 063526 (2005), hep-th/0410230.

[55] S. H. S. Alexander (2006), hep-th/0601034.

[56] D. Guarrera and A. J. Hariton, Phys. Rev. D76, 044011 (2007), gr-qc/0702029.

[57] S. Alexander and N. Yunes, Phys. Rev. Lett. 99, 241101 (2007), hep-th/0703265.

[58] S. Alexander and N. Yunes, Phys. Rev. D75, 124022 (2007), 0704.0299.

[59] K. Konno, T. Matsuyama, and S. Tanda, Phys. Rev. D76, 024009 (2007), arXiv:0706.3080 [gr-qc].

[60] S. Alexander, L. S. Finn, and N. Yunes, Phys. Rev. D78, 066005 (2008), 0712.2542.

[61] S. H. Alexander, M. E. Peskin, and M. M. Sheikh-Jabbari (2007), hep-ph/0701139.

[62] N. Yunes and C. F. Sopuerta, Phys. Rev. D77, 064007 (2008), 0712.1028.

[63] T. L. Smith, A. L. Erickcek, R. R. Caldwell, and M. Kamionkowski, Phys. Rev. D77, 024015 (2008), 0708.0001

[64] D. Grumiller and N. Yunes, Phys. Rev. D77, 044015 (2008), 0711.1868.

[65] D. Grumiller, R. Mann, and R. McNees, Phys. Rev. D78, 081502 (2008), 0803.1485.

[66] N. Yunes and L. S. Finn, J. Phys. Conf. Ser. 154, 012041 (2009), 0811.0181.

[67] M. B. Cantcheff, Phys. Rev. D78, 025002 (2008), 0801.0067.

[68] S. Alexander and N. Yunes, Phys. Rev. D77, 124040 (2008), 0804.1797.

[69] K. Konno, T. Matsuyama, and S. Tanda (2009), 0902.4767.

[70] C. F. Sopuerta and N. Yunes, Phys. Rev. D80, 064006 (2009), 0904.4501.

[71] N. Yunes and C. F. Sopuerta (2009), 0909.3636.

[72] D. Psaltis, D. Perrodin, K. R. Dienes, and I. Mocioiu, Phys. Rev. Lett. 100, 091101 (2008), 0710.4564.

[73] D. Psaltis (2008), 0806.1531.

[74] S. A. Story, P. L. Gonthier, and A. K. Harding, Astrophys. J. 671, 713 (2007), 0706.3041.

[75] A. A. Abdo, M. Ackermann, M. Ajello, W. B. Atwood, M. Axelsson, L. Baldini, J. Ballet, G. Barbiellini, M. G. Baring, D. Bastieri, et al., Science 325, 848 (2009).

[76] S. M. Ransom, P. Ray, F. Camilo, and M. Roberts, in Bulletin of the American Astronomical Society (2010), vol. 41 of Bulletin of the American Astronomical Society, 
pp. $464-+$.

[77] C. Faucher-Giguère and A. Loeb, Journal of Cosmology and Astro-Particle Physics 1, 5 (2010), 0904.3102.

[78] J. M. Lattimer and B. F. Schutz, Astrophys. J. 629, 979 (2005), arXiv:astro-ph/0411470.

[79] M. Kramer and N. Wex, Class. Quant. Grav. 26, 073001 (2009).

[80] C. W. Misner, K. Thorne, and J. A. Wheeler, Gravitation (W. H. Freeman \& Co., San Francisco, 1973).

[81] S. Alexander, L. S. Finn, and N. Yunes, Phys. Rev. D 78, 066005 (2008), 0712.2542.

[82] N. Yunes and D. N. Spergel, Phys. Rev. D80, 042004 (2009), 0810.5541.

[83] X. Jaen, J. Llosa, and A. Molina, Phys. Rev. D34, 2302 (1986).

[84] D. A. Eliezer and R. P. Woodard, Nucl. Phys. B325, 389 (1989).

[85] J. Z. Simon, Phys. Rev. D41, 3720 (1990).

[86] S. DeDeo and D. Psaltis, Phys. Rev. D78, 064013 (2008), 0712.3939

[87] A. Cooney, S. DeDeo, and D. Psaltis, Phys. Rev. D79,
044033 (2009), 0811.3635.

[88] C. M. Bender and S. A. Orszag, Advanced Mathematical Methods for Scientists and Engineers (McGraw-Hill, New York, 1978).

[89] J. B. Hartle, Astrophys. J. 150, 1005 (1967).

[90] B. Friedman and V. R. Pandharipande, Nucl. Phys. A361, 502 (1981).

[91] D. Blaschke, N. K. Glendenning, and A. Sedrakian, eds., Physics of Neutron Star Interiors, vol. 578 of Lecture Notes in Physics, Berlin Springer Verlag (2001).

[92] V. Kalogera and D. Psaltis, Phys. Rev. D61, 024009 (2000), astro-ph/9903415.

[93] GRTensorII, this is a package which runs within Maple but distinct from packages distributed with Maple. It is distributed freely on the World-Wide-Web from the address: http://grtensor.org.

[94] P. O. Mazur and E. Mottola (2001), gr-qc/0109035.

[95] M. Visser and D. L. Wiltshire, Class. Quant. Grav. 21, 1135 (2004), gr-qc/0310107. 